\title{
Efficient, Semi-Transparent Neutral-Coloured Solar Cells Based on Microstructured Formamidinium Lead Trihalide Perovskite
}

Giles E. Eperon, Daniel Bryant, Joel Troughton, Samuel D. Stranks, Michael B. Johnston, Trystan Watson, David A. Worsley and Henry J. Snaith*

G. E. Eperon, S. D. Stranks, Prof. M. B. Johnston, Prof. H. J. Snaith

Department of Physics, University of Oxford, Clarendon Laboratory, Parks Road, Oxford OX1 3PU, UK

E-mail: h.snaith1@physics.ox.ac.uk

Dr D. Bryant, J. Troughton, Dr T. Watson, Prof. D. Worsley

Specific, College of Engineering, Swansea University, Baglan Bay Innovation, Knowledge Centre, Central Avenue, Baglan SA12 7AX, UK

\section{Table of Contents figure}

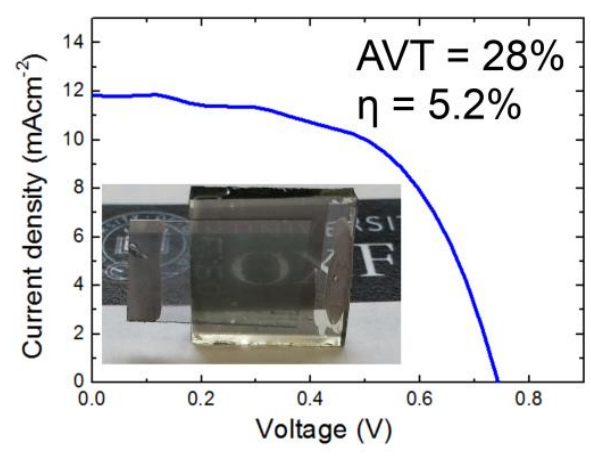

Keywords: perovskite, solar cell, semi-transparent, building-integrated photovoltaics, neutral colour 


\begin{abstract}
Efficient, neutral-coloured semitransparent solar cells are of commercial interest for incorporation into the windows and surfaces of buildings and automobiles. Here, we report on semitransparent perovskite solar cells that are both efficient and neutral-coloured, even in full working devices. Using the microstructured architecture previously developed, we achieve higher efficiencies by replacing methylammonium lead iodide perovskite with formamidinium lead iodide. Current-voltage hysteresis is also much reduced. Furthermore, we apply a novel transparent cathode to the devices, enabling us to fabricate neutral-coloured semitransparent full solar cells for the first time. Such devices demonstrate over $5 \%$ power conversion efficiency for average visible transparencies of almost $30 \%$, retaining impressive colour-neutrality. This makes these devices the best-performing single junction neutralcoloured semitransparent solar cells to date. These microstructured perovskite solar cells are shown to have a significant advantage over silicon solar cells in terms of performance at high incident angles of sunlight, making them ideal for building integration.
\end{abstract}


Semitransparent solar cells are an attractive concept for enabling building-integrated power generation. Enabling power to be generated from surfaces such as facades and windows opens up much greater surface area for solar generation. For a semi-transparent solar cell, there is an obvious trade-off between capturing light for solar energy generation, and allowing light through a window. This is quantified in terms of power conversion efficiency (PCE) versus average visible transmittance (AVT). Additionally, further compromises are often necessary to enable functional devices to be semitransparent. The necessity for transparent active layers and electrodes means that many devices are forced to compromise on efficiency, since these often do not function as well as their opaque counterparts. The most common compromise, however, is on colouring. The most desired tint for a window is neutral-coloured, but many semi-transparent solar cells developed thus far are not neutralcoloured. Thus there still exists a necessity for a neutral-coloured, efficient semi-transparent solar cell for building-integated photovoltaics.

Recently, there has been a flurry of attention in the solar cell community focussed on the emerging photovoltaic material of hybrid organic-inorganic metal halide perovskites. These materials, based generally on hybrid organic-inorganics forming the $A B X_{3}$ perovskite structure, show a suitable bandgap, high absorption coefficient and long diffusion lengths, making them ideal as an absorber in photovoltaic devices. ${ }^{1-4}$ Recently, power conversion efficiencies (PCEs) of over $19 \%$ have been reported, compared to just $3.8 \%$ in 2009 , making them the most quickly advancing solar technology yet. ${ }^{5-9}$ Previously, we have reported on the application of the most commonly studied $\mathrm{MAPbl}_{3}$ perovskite in neutral-coloured semitransparent solar cells for building-integrated photovoltaics. ${ }^{10}$ Spontaneous dewetting of an excessorganic containing perovskite precursor dissolved in a low vapour pressure solvent enabled the formation of a microstructured array of perovskite "islands", which were able to generate respectable efficiencies despite comprising a discontinuous planar heterojunction architecture. The combination of fully absorbing islands and transparent regions allowed such films to be neutral-coloured and semi- 
transparent, with up to nearly $60 \%$ active layer average visible transmittance (defined as the mean transmittance of the film between $370 \mathrm{~nm}$ and $740 \mathrm{~nm})$. However, in this first report of semi-transparent perovskite solar cells, we were unable to employ a sufficiently transparent cathode that would retain the neutral colouration. In full devices, efficiencies of $\sim 5 \%$ were achieved for an average visible transmittance of $20 \%$; however, this was with a semi-transparent gold cathode, which no longer provided neutral colour. We concluded that the gold cathode was the limiting factor - with a more transparent cathode we should achieve better AVTs and also retain neutral colouration. More recently, others have reported on a semitransparent perovskite solar cell also employing a thin gold cathode, with 6.4\% PCE and 30\% full device AVT. However, they employ a thin but continuous layer of perovskite, giving a brown-yellow device. ${ }^{11}$

Other technologies have also achieved high efficiencies per transparency. Organic photovoltaics, which have shown promise with good efficiencies per transparency, often compromise on colour-neutrality. It is difficult with this technology to obtain a spectrally flat absorption across the whole visible spectrum with specifically tailored polymers, especially without losing efficiency accordingly. Additionally, the organic photovoltaic technology involves an inherent voltage loss associated with energy required to dissociate tightly bound excitons into free electrons and holes in the device, whereas the perovskite, in which excitons are easily dissociated, does not exhibit such a loss. ${ }^{12-14}$ This suggests an ultimate advantage for the semiconducting perovskites. Despite these setbacks, single-junction organic photovoltaics with $4.2 \%$ efficiency for $30 \%$ transparency have been reported ${ }^{15}$, and organic tandem devices have very recently have demonstrated an impressive $7.9 \%$ efficiency for $40 \%$ transparency in the blue-green region of the visible spectrum. ${ }^{16,17}$ However, such devices again compromise on colour; they are not properly neutral-coloured. Moreover, the cost of fabricating such specifically tailored polymers is likely to be a real challenge and a barrier to market entry before full scale up can be achieved. Tandem devices in particular are complex to fabricate and require many carefully processed layers. The 
perovskite materials, however, are extremely cheap to synthesise and fabricate devices from, meaning that scale-up should be much more commercially attractive and will present a cost-effective option when considering the module payback times. Most processing can also take place at low temperatures, with no need for vacuum processing. Whilst most work has focused on the methylammonium lead iodide perovskite, $\mathrm{CH}_{3} \mathrm{NH}_{3} \mathrm{Pbl}_{3}$ (we note that while significant effort has focussed on the mixed halide precursor, $\mathrm{CH}_{3} \mathrm{NH}_{3} \mathrm{I}_{3-\mathrm{x}} \mathrm{Cl}_{\mathrm{x}}$, recent reports suggest that the chloride content in the final product is low, so we include this material as $\mathrm{CH}_{3} \mathrm{NH}_{3} \mathrm{Pbl}_{3}$ here too ${ }^{18-22}$, more recently several groups have demonstrated that the methylammonium $\left(\mathrm{CH}_{3} \mathrm{NH}_{3}{ }^{+}\right.$, hereafter $\left.\mathrm{MA}\right)$ component can be replaced with formamidinium $\left(\mathrm{HC}\left(\mathrm{NH}_{2}\right)_{2}{ }^{+}\right.$, hereafter $\left.\mathrm{FA}\right)$, resulting in a slightly larger lead iodide-based crystal lattice, with the effect of narrowing the bandgap closer to that optimal for solar cells, from $1.57 \mathrm{eV}$ to $1.48 \mathrm{eV}$, $^{7,23,24}$ Moreover, it has been observed that the $\mathrm{FAPbl}_{3}$ perovskite is more thermally stable at raised temperature, more photostable under continual operation, and that it does not undergo a phase transition in the solar cell operating regime, which could be an issue for the $\mathrm{MAPbl}_{3}$ perovskite. ${ }^{7,23}$ As such, the $\mathrm{FAPbl}_{3}$ perovskite has a number of highly advantageous characteristics for its use in solar cells.

Herein, we report on two major improvements to the semitransparent perovskite solar cell. We replace $\mathrm{MAPbl}_{3}$ with the advantageous $\mathrm{FAPbl}_{3}$ in the microstructured architecture, which allows us to achieve significantly higher efficiencies for equivalent transparencies. Such devices display very little J-V hysteresis, and have high stabilised power output. Secondly, we employ a novel ITO and precious metalfree transparent cathode in such devices, allowing fully operating, completely neutral coloured semitransparent, perovskite solar cells for the first time.

We fabricated $\mathrm{FAPb}_{3}$ in the microstructured architecture by using a precursor containing excess of organic components, and in a low vapour pressure solvent, dimethylsulfoxide (DMSO). This combination was previously found to produce a regular array of perovskite islands for $\mathrm{MAPbl}_{3}{ }^{10}$ It is thought that the 
presence of the excess organic results in slower crystallisation, as a long anneal is required to fully remove the excess organic. ${ }^{20}$ Having a low vapour pressure solvent means that the perovskite starts to crystallise while the solvent is still present, and the combination of slow crystallisation and slow solvent removal results in the perovskite nucleating in a discontinuous island-type structure. The dynamics are more properly understood with reference to a mathematical model for this behaviour which we have previously reported. ${ }^{25}$ In the case of the organic excess MA perovskite previously studied, lead chloride is used as the lead source, and MAI as the MA and I source. It is assumed that the reaction progresses thusly: ${ }^{26}$

$3 \mathrm{MAl}+\mathrm{PbCl}_{2}->\mathrm{MAPbl}_{3}+2 \mathrm{MACl}$

The $\mathrm{MAPbl}_{3}$ is the perovskite formed, and the $\mathrm{MACl}$ is predominantly removed via sublimation or degradation, which is experimentally observed to occur at temperatures greater than $80^{\circ} \mathrm{C}^{26,27}$. In the case of the FA however, because the FA cation is larger, it is not so easily removed. We attempted to produce similarly structured films in this manner, but were unsuccessful and the films did not result in the same morphology - they were very rough, discontinuous, and non-uniform. Likewise, using a stoichiometric 1:1 FAl: $\mathrm{Pbl}_{2}$ precursor in DMSO also produced very rough, non-uniform films. It appears that the excess organic is critical to obtain the microstructuring. As such, we took a new route to obtain microstructured films of $\mathrm{FAPbl}_{3}$ : we incorporated MA as a sacrificial component. We assume that the reaction progress is as shown below:

$2 \mathrm{MAl}+\mathrm{FAl}+\mathrm{PbCl}_{2}->\mathrm{FAPbl}_{3}+2 \mathrm{MACl}$ 
Since $\mathrm{FACl}$, being a larger molecule than $\mathrm{MACl}$, requires higher temperatures to sublime than $\mathrm{MACl}$, the $\mathrm{MACl}$ will be removed on heating at $>80^{\circ} \mathrm{C}$, leaving $\mathrm{FAPbl}_{3}$ behind. We fabricated films of this material by dissolving the precursors in DMSO, spin-coating in a single step, and annealing first at $130^{\circ} \mathrm{C}$ for 20 minutes and subsequently at $170^{\circ} \mathrm{C}$ for 10 minutes. The first step is to remove the excess $\mathrm{MACl}$ in a similar manner to our previous report, ${ }^{10}$ and the second step is to force the $\mathrm{FAPbl}_{3}$ into its black, rather than yellow, phase, as previously reported. ${ }^{7,23}$ Films formed in this manner did indeed produce a material with a somewhat similar microstructuring as was observed in the $\mathrm{MAPbl}_{3}$ material, as we show in Figure 1.
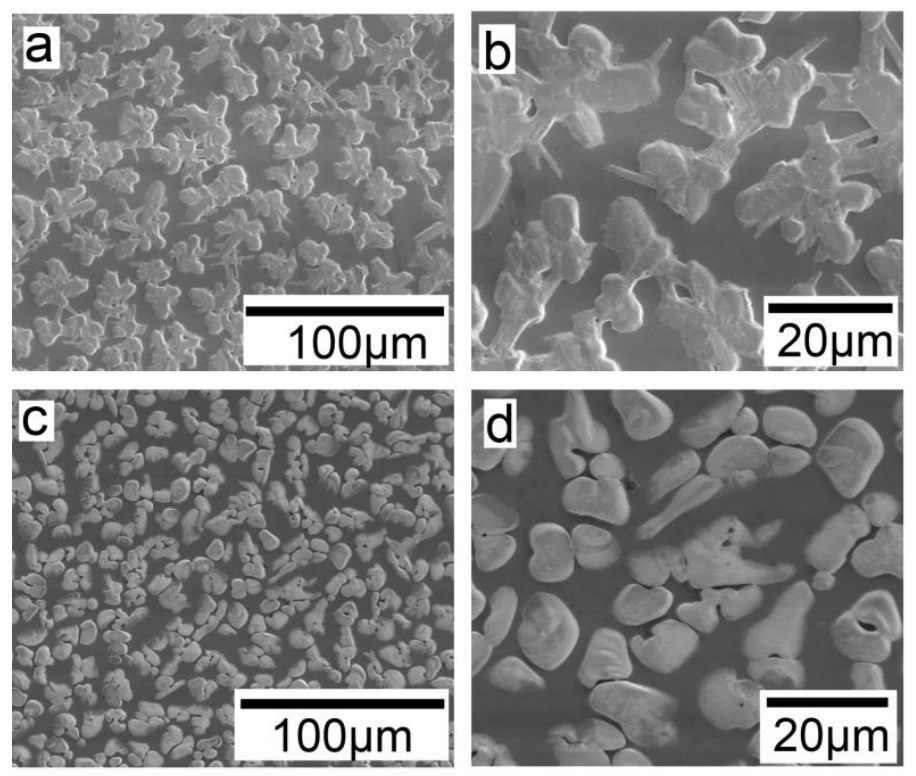

Figure 1. a) and b): different magnifications of FA-containing microstructured perovskite films produced from the precursors detailed in equation (1) and annealed first at $130^{\circ} \mathrm{C}$ and then at $170^{\circ} \mathrm{C} . \mathrm{c}$ ) and d): MA-containing films produced from the precursors detailed in (2) and annealed at $130^{\circ} \mathrm{C}$.

In order to confirm that the material produced is $\mathrm{FAPbl}_{3}$, we carried out X-ray diffraction (XRD) measurements on the films, as shown in Figure 2. 

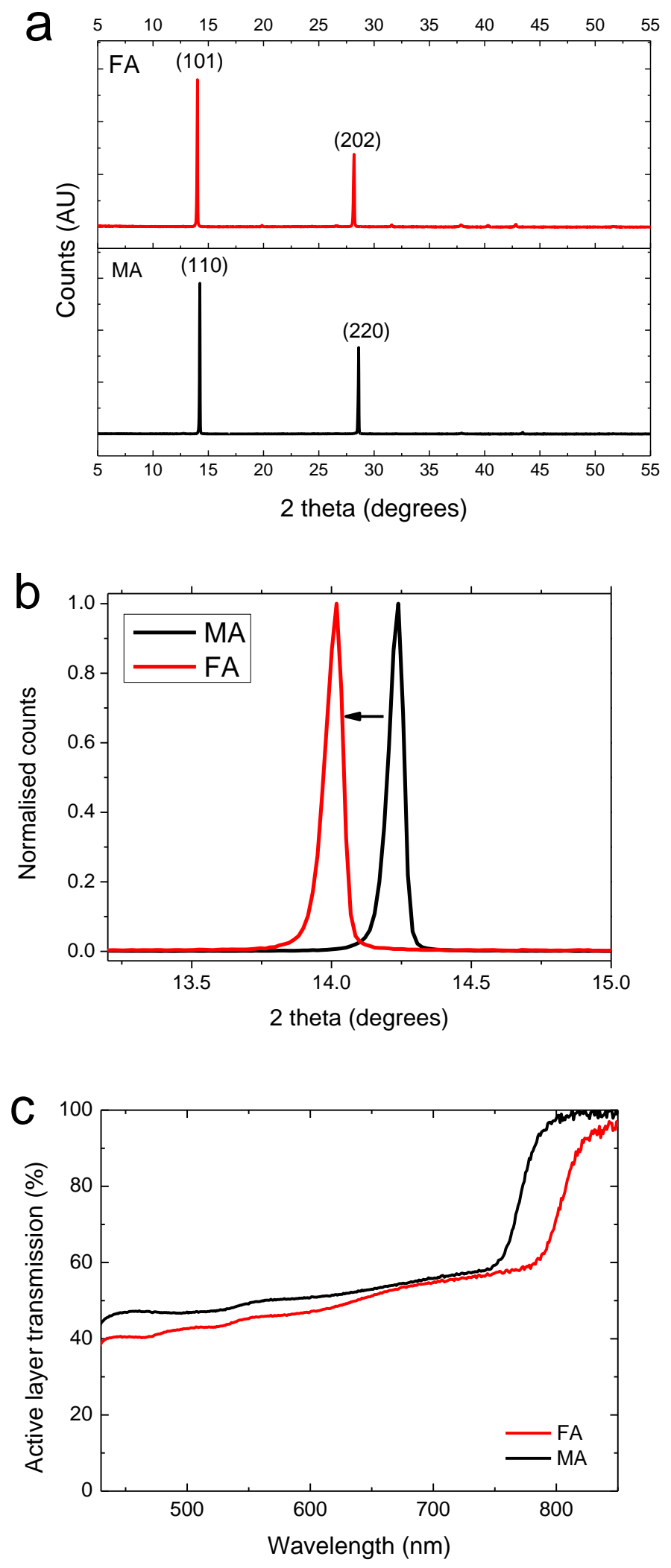
Figure 2. a) XRD spectra of the FA and MA films. Labeled peaks correspond to those indexed to the literature crystal structures of a tetragonal strcture with $a=8.64 \AA, c=12.64 \AA$ for $\mathrm{MAPbl}_{3}$ and trigonal structure with $a=8.99 \AA, c=11.0 \AA$ for $F A P b I_{3}$. b) Zoom of the peaks at $\sim 14^{\circ}$ in the XRD spectra showing the shift from MA to FA. c) Transmittance spectra of the two materials. The data here shows the transmittance of the active layer, including spiro-OMeTAD and the titania compact layer.

We observe a material with highly oriented crystallinity in the FA material, similar to that observed in the MA material. The peaks observed can be assigned to the $(110) /(101)$ and $(220) /(202)$ peaks of a tetragonal/trigonal lattice structure (with $\alpha=\beta=\gamma=90^{\circ}$ for the tetragonal, $\alpha=90^{\circ}, \beta=90^{\circ}, \gamma=120^{\circ}$ for the trigonal structure) respectively; the MA material agrees well with previous reports of a tetragonal material with $a=8.64 \AA, c=12.64 \AA$, and we observe an obvious shift in $2 \theta$ for the FA material (as shown for the peak at $\sim 14^{\circ}$ in Figure $2 b$ ), giving good agreement to a trigonal material with $a=8.99 \AA, c=11.0 \AA$, as previously reported for the black phase of $\mathrm{FAPbl}_{3}{ }^{7,23,28,29}$ No peaks corresponding to $\mathrm{MAPbl}_{3}$ are seen in the FA material's spectrum, allowing us to assign it unambiguously as almost pure black phase $\mathrm{FAPbl}_{3}$. To further confirm the identity of the material, and to quantify the transparency, we measured the transmittance of the materials, as shown in Figure $2 \mathrm{c}$ ). We note that since we are interested in their use in semitransparent solar cells, we take spectra of the whole active layer of such a device, which is compact $\mathrm{TiO}_{2}$ /perovskite/2,2',7,7'-tetrakis( $\mathrm{N}, \mathrm{N}^{\prime}$-di-p-methoxyphenylamine)-9,9'-spirobifluorene (spiroOMeTAD). The method used to obtain the active layer transmittance is discussed in detail elsewhere. ${ }^{10}$ We observe that whilst the MA material absorbs out to $790 \mathrm{~nm}$, in accordance with a bandgap of $\sim 1.57 \mathrm{eV}$, the FA material absorbs out to $\sim 840 \mathrm{~nm}$, in good agreement with previous reports of a bandgap of $\sim 1.48 \mathrm{eV}^{23}$ We thus can confirm that the material is predominantly $\mathrm{FAPbl}_{3}$; if there is any MA left, it has a negligible effect on the crystal structure and absorption spectrum. Furthermore, the FA material displays a quite flat transmission spectrum across the visible range, indicating that it is likely similarly 
colour-neutral to the MA material. Both materials formed in this microstructured way display a high transmittance, which is highly encouraging for their use in semitransparent solar cells.

Having confirmed that we have been able to fabricate semitransparent microstructured arrays of $\mathrm{FAPbl}_{3}$ islands, we fabricated planar heterojunction solar cells from such films, with the device structure shown in Figure 3a). 

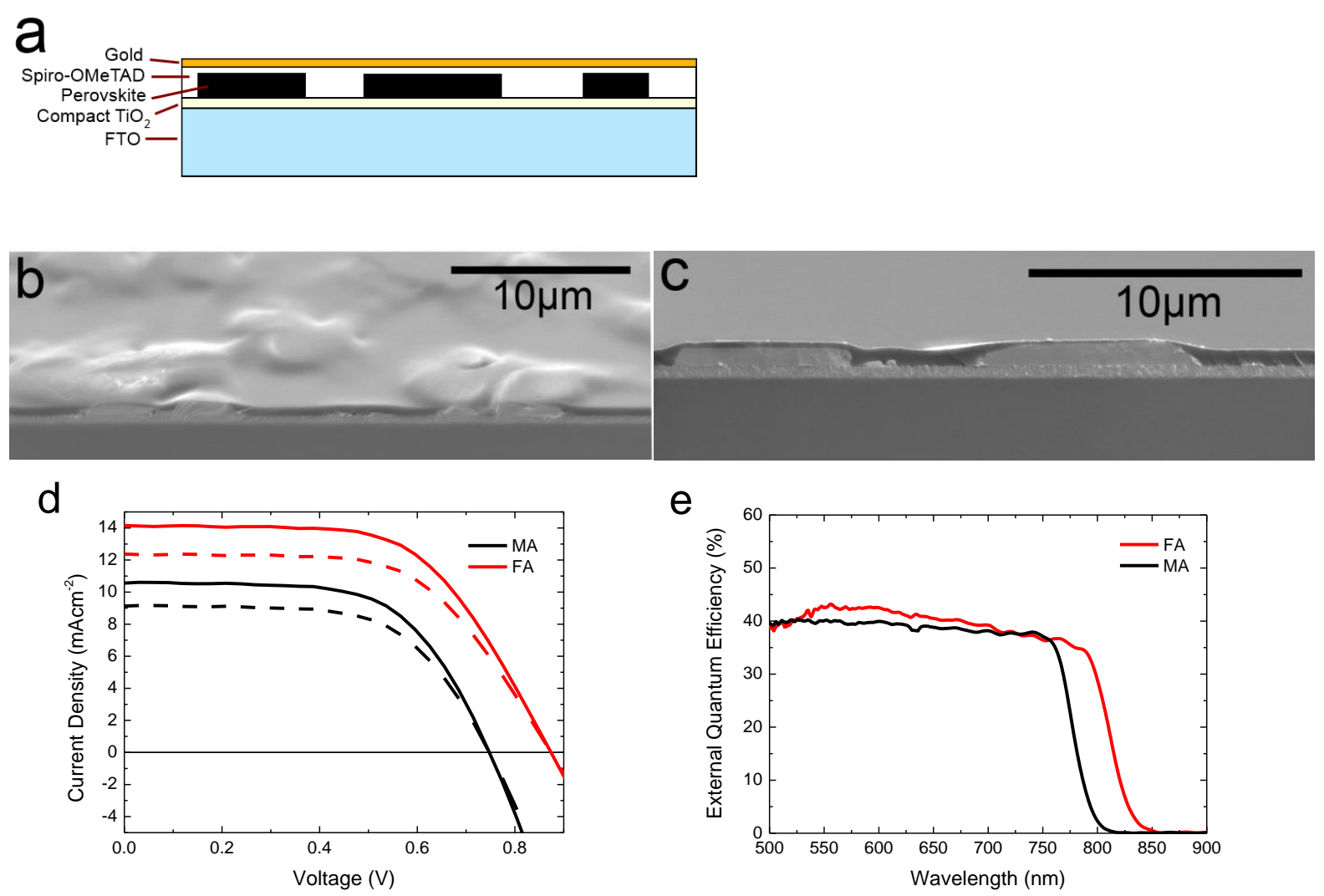

e
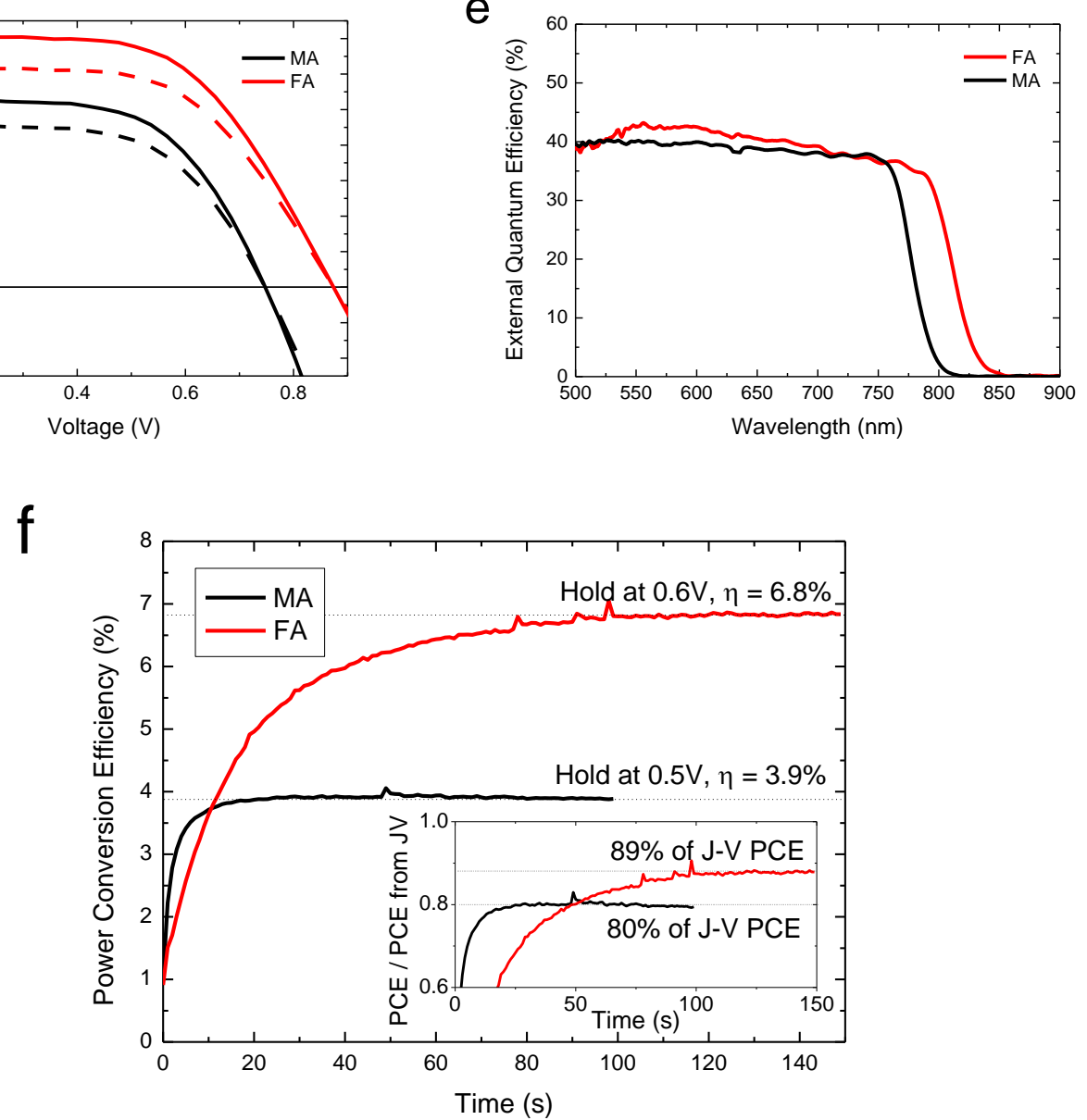

Figure 3. a) Diagram of the device architecture employed. b) Tilted and c) cross-sectional SEMs of a full semitransparent $\mathrm{FAPbl}_{3}$ solar cell showing the islands of perovskite coated with spiro-OMeTAD. d) J-V characteristics of $\mathrm{MAPbl}_{3}$ and $\mathrm{FAPbl}_{3}$ solar cells, measured under simulated $100 \mathrm{mWcm} \mathrm{AM}^{-2} .5$ illumination, for both the measured data (solid lines) and that calculated for just one light pass (dashed 
lines). e) EQE spectra for representative MA and FAPbl 3 solar cells. f) PCE extracted at maximum power point under $100 \mathrm{mWcm}^{-2}$ illumination over time, and in the inset, the same measurement plotted as a fraction of the PCE determined via a fast J-V scan.

Previously, we confirmed that due to the rectifying nature of the titania-Spiro-OMeTAD contact, such discontinuous devices are still impressively functional. ${ }^{10}$ We show cross-sectional SEM images of such devices in Figure $3 b$ ); we clearly observe the perovskite islands of $\sim 900 \mathrm{~nm}$ thickness, covered by a thin layer of spiro-OMeTAD. In order to assess comparative performance of the FA films compared to MA films, we used thick gold electrodes as the cathode. Although transparency is then reduced, we did not wish device performance to be limited by the electrode initially. We show the current-voltage characteristics measured under simulated sunlight $\left(A M 1.5,100 \mathrm{mWcm}^{-2}\right)$ illumination from the bestperforming devices fabricated with each material in Figure 3c), with full device parameters extracted shown in Table 1.

\begin{tabular}{lllllll}
\hline & Jsc $\left(\mathrm{mAcm}^{-2}\right)$ & $\mathbf{V}_{\text {oc }}(\mathrm{V})$ & $\mathbf{F F}$ & $\mathbf{\eta}(\%)$ & $\begin{array}{l}\text { AVT (active } \\
\text { layer) (\%) }\end{array}$ & $\boldsymbol{\eta}_{\text {MPP }}(\%)$ \\
\hline FA & 14.2 & 0.86 & 0.60 & 7.4 & 33.6 & 6.8 \\
FA (1 pass) & 12.4 & & & 6.4 & & \\
MA & 10.6 & 0.64 & 0.54 & 4.9 & 40.5 & 3.9 \\
MA (1 pass) & 9.1 & & & 4.2 & & \\
\hline
\end{tabular}

Table 1. Table showing device characteristics of best $\mathrm{FAPbl}_{3}$ and $\mathrm{MAPbl}_{3}$-based semi-transparent solar cells fabricated with gold contacts. The data for 1 pass refers to the calculated corrected current from the same devices if light was not absorbed in the second pass reflected from the gold electrode, which is the data that would be important for a totally semi-transparent solar cell.

We show data for both two passes of light (as measured due to the reflection from the gold contact) and for one pass of light (the best efficiency possible for such a device if it had a perfect transparent 
cathode, calculated as described elsewhere $\left.{ }^{10}\right)$. We show the statistics for the whole batch fabricated in the SI. We note that the AVT of the FA films is a little lower than the MA films $(33.6 \%$ compared to 40.5\%), and furthermore the $\mathrm{FAPbl}_{3}$ provides absorption over a greater fraction of the solar spectrum due to its reduced bandgap. Accordingly, we see that the photocurrent generated is higher than for the $\mathrm{MAPbl}_{3}$. More surprising is the increase in open-circuit voltage $\left(\mathrm{V}_{\mathrm{oc}}\right)$ that we observed with the $\mathrm{FAPbl}_{3}$. Due to its reduced bandgap, we would expect that if anything, the $V_{\text {oc }}$ would decrease. We have experimentally observed that this increased voltage is reproducible, and its origin is currently under further investigation. It is possible that the higher-temperature anneal is beneficial to the compact titania, and allows for higher oxygen-doping. This would then raise the turn-on voltage of a spiro-titania diode; any contact between spiro-OMeTAD in the cell will act as a parallel shunt diode, so increasing the turn-on voltage of these diodes would have the effect of overall reducing the detrimental effect on $\mathrm{V}_{\text {oc }}$ that this normally has. ${ }^{10}$ The impact of the increased current and voltage in the $\mathrm{FAPbl}_{3}$ compared to the $\mathrm{MAPbl}_{3}$ is significant; power conversion efficiency increases from $4.9 \%$ to $7.4 \%$ for the best devices ( $2.8 \%$ to $4.3 \%$ on average across the batch).

We show the EQE of representative MA and FA cells in Figure 3d). We observe that the FA generates photocurrent up to $\sim 840 \mathrm{~nm}$, and the MA up to $\sim 790 \mathrm{~nm}$, in good agreement with the absorption spectra for the different perovskite materials.

It has been observed recently that such planar heterojunction perovskite solar cells are likely to exhibit hysteresis in their J-V curves. ${ }^{30}$ This can lead to exaggerated PCEs based on fast J-V scans; as such, it is vital to also measure the stabilised power output of such devices. It has been suggested that $\mathrm{FAPbl}_{3}$ might display less hysteresis compared to $\mathrm{MAPbl}_{3}$, in which case its stabilised power output should be closer to its fast J-V PCE. ${ }^{7}$ To assess this, as shown in Figure 3e) and 3f), we measured the power output over time when holding the devices at their maximum power point as determined by the fast J-V scan. We plot both the absolute PCE generated, and PCE as a fraction of that determined by the fast J-V fast. 
We observe that the $\mathrm{MAPbl}_{3}$ semitransparent cell stabilises quickly, but only at $3.9 \%$, or $80 \%$ of its PCE according to the fast J-V scan. The $\mathrm{FAPbl}_{3}$ cell takes longer to stabilise, but it then generates $6.8 \% \mathrm{PCE}$, which is $89 \%$ of its fast J-V scan efficiency. These results are very promising for the $\mathrm{FAPbl}_{3}$; they suggest that not only are the stabilised efficiencies significantly higher than a similarly transparent $\mathrm{MAPbl}_{3}$, but also they exhibit a less severe hysteretic effect, meaning that the PCE extracted from a fast J-V scan is closer to the stabilised PCE.

Having demonstrated that incorporating $\mathrm{FAPbl}_{3}$ into the semitransparent microstructured perovksite solar cell architecture can lead to significantly enhanced efficiencies, we then move on to solve the remaining challenge for semitransparent perovskite solar cells; making the entire device fully semitransparent, whilst retaining the neutral colouration. Whilst we have a reasonable transparent anode in the FTO-coated glass (hereafter just FTO), the cathode is more challenging. Previously, we have relied on a thin gold contact as the semitransparent contact, but this has a detrimental effect on the transparency and neutral colouration. Recently, we have developed a transparent conductive adhesive laminate electrode based on a nickel mesh, which can be laminated onto the solar cell via application of a PEDOT:PSS-based pressure-activated conductive adhesive. This is described in greater detail elsewhere. ${ }^{31}$ It provides a low-cost, transparent electrode with good colour-neutrality, and has been shown to be capable of replacing gold as an effective cathode in perovskite solar cells. It comprises a commercial nickel mesh embedded in a PET film, onto which a transparent conductive adhesive comprising a mixture of PEDOT:PSS and an acrylic glue is coated. This electrode is then pressurelaminated onto the solar cells at room temperature, after having spray-coated a thin layer of PEDOT:PSS on top of the spiro-OMeTAD of the solar cells. We apply this transparent laminated cathode (TLC) to the $\mathrm{FAPbl}_{3}$ semitransparent solar cells, to achieve fully semitransparent working perovskite solar cells with neutral colour for the first time. 
a

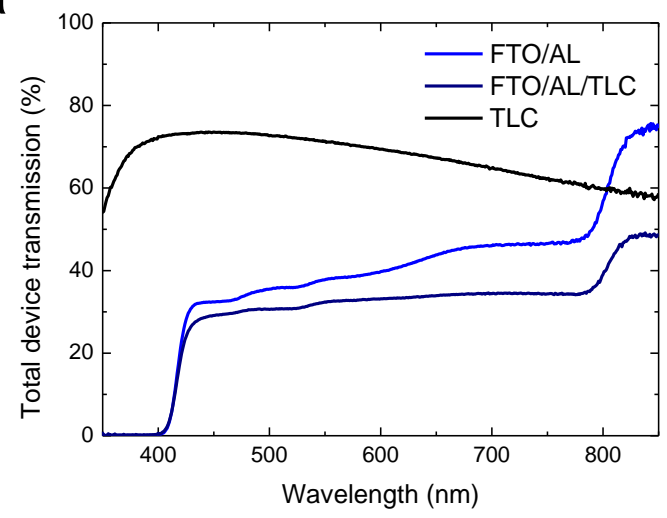

b
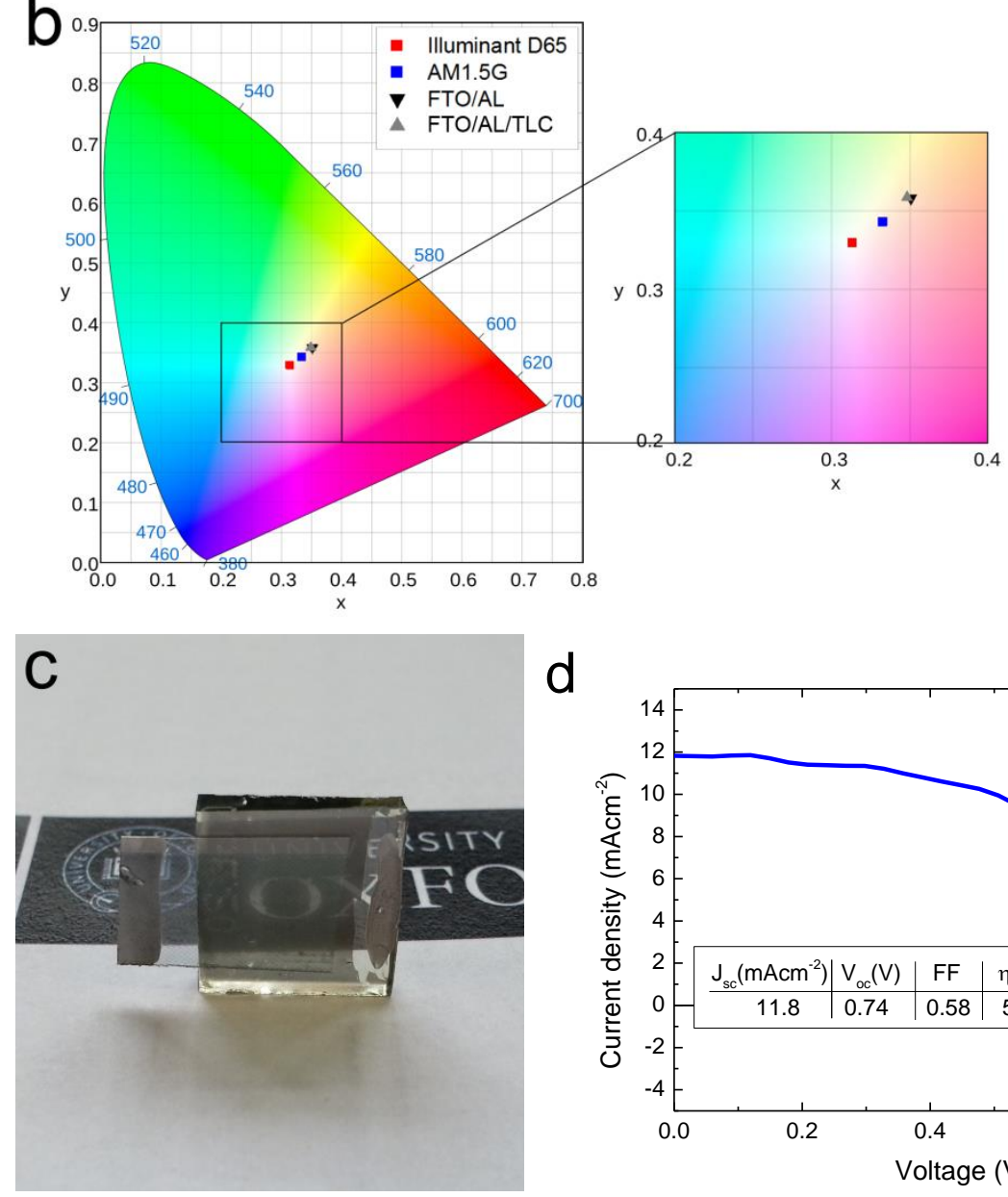

d

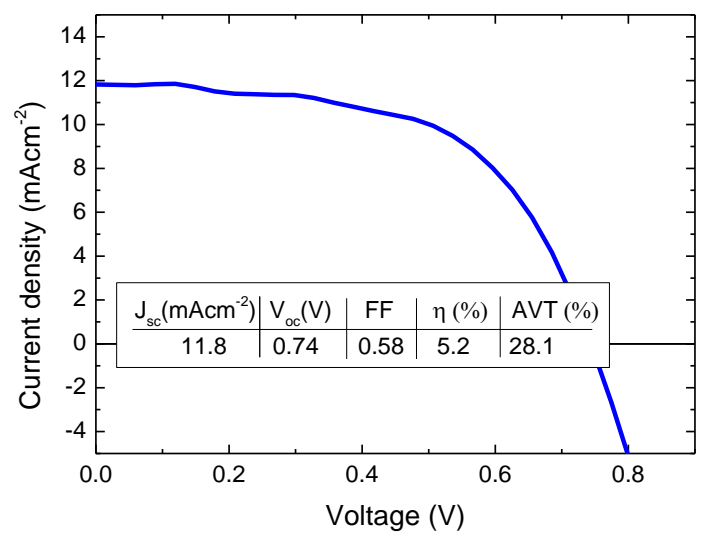

Figure 4. a) Transmission spectra of the structures FTO/Active layer, TLC on glass, and FTO/Active layer/TLC. b) colour coordinates of the films with transmittance spectra shown in (a) under AM1.5 illumination, on the CIE xy 1931 chromacity diagram, and the enlarged central region. Colour coordinates of the D65 standard daylight illuminant and AM1.5 illumination are also shown. c) Photograph of a fully 
semi-transparent $\mathrm{FAPbl}_{3}$ device including the laminated transparent cathode d) Current-voltage characteristics of a fully semitransparent device such as depicted above. (University of Oxford logo reproduced with permission. Courtesy of University of Oxford.)

In Figure 4a), we show the transmission of the TLC alone, and the transmission of a full $\mathrm{FAPbl}_{3}$ solar cell before $(\mathrm{FTO} / \mathrm{AL}$, where $\mathrm{AL}=$ active layer) and after the application of the TLC (FTO/AL/TLC). We observe that the average visible transmittance is reduced from $34.2 \%$ to $28.1 \%$ upon application of the cathode. The flat transmission spectrum is retained, even enhanced. We note that the TLC on its own attenuates much more light than the increased attenuation when applied upon the perovskite cell. We assume that this is due to a large fraction of the attenuation with the TLC being reflection rather than absorption, which is predominantly absent at the AL/TLC interface in the complete stack. We verify the colourneutrality of the whole device by calculating the colour perception indices according to the CIE 1931 xy colour space standard. Transmitted light is represented by the product of the AM1.5 spectrum and the transmission of the sample in question. In Figure 4b), we plot the colour coordinates of the active layer only (compact titania/perovskite/spiro-OMeTAD), the active layer on FTO-glass, and the whole FTO/AL/TLC device, compared to the reference daylight illuminant D65 and the AM1.5 spectrum. We observe that the films have excellent colour-neutrality, lying well within the central region of the chromacity diagram, close to the AM1.5 spectrum. Application of the transparent electrodes does not affect the colour perception to any great extent, demonstrating that they are a suitable choice of transparent conductive electrode for the $\mathrm{FAPbl}_{3}$ semitransparent solar cell. We show a photograph of a neutral-coloured semitransparent $\mathrm{FAPbl}_{3}$ device with both electrodes in Figure 4c), exhibiting good visual transparency. In Figure 4d) we show the current-voltage characteristics of the best-performing fully semi-transparent FTO/AL/TLC FAPbl ${ }_{3}$ device fabricated. It displays a lower short-circuit current and open-circuit voltage than the equivalent devices with gold electrodes, though the current density is 
close to as expected with 1 pass of light. Accordingly, we obtained a PCE of 5.2\%, for a neutral-coloured semitransparent solar cell with an AVT of $28 \%$, with an active area of approximately $0.5 \mathrm{~cm}^{-2}$, masked to $0.0625 \mathrm{~cm}^{2}$.

A neutral-coloured perovskite device with $5.2 \%$ efficiency and complete device semitransparency of almost $30 \%$ represents a significant step towards the commercialisation of this technology. The efficiency/transparency ratio is almost as good as some of the recently reported single junction organic solar cells, despite the fact that the organic solar cell field is more than 20 years old. ${ }^{15,16,32}$ These characteristics in fact make this one of the best performing neutral-coloured single junction semitransparent solar cells reported. However, the key advantage that the cells realised here have is in cost. The perovskite active material itself is comprised of readily available, cheap materials. Furthermore, herein we have demonstrated the possibility of replacing an expensive precious metal cathode with a cheap, and simple to process nickel-based mesh. Neither is there any ITO in the device, a common concern due to the limited availability of indium. Fabrication of the whole device takes place via solution-processing at low temperatures and at atmospheric pressure; not a single vacuum process is required. This results in a semi-transparent solar cell which is extremely cheap, and the cost of coating such devices onto window glass would likely be less than the cost of the glass itself. ${ }^{33}$ The limiting factors in the cost of the device now become the use of spiro-OMeTAD as HTM and the high-temperature annealing for the titania compact layer. Replacement of spiro-OMeTAD would also be advantageous for the AVT of such devices, as spiro-OMeTAD absorbs strongly below 430nm, affecting the AVT somewhat. The titania compact layer is annealed at $500^{\circ} \mathrm{C}$, increasing the processing cost incurred in device production. Neither of these problems are insoluble however, and work in the perovskite solar cell community is well focussed on overcoming these limitations. ${ }^{34-37}$

It is observed that the efficiency of the $\mathrm{FAPbl}_{3}$ devices drop upon use of the TLC. This mainly stems from a loss in $\mathrm{V}_{\mathrm{oc}}$ and $\mathrm{FF}$, which is possibly due to an increase in series resistance from the thick 
PEDOT:PSS/adhesive blend, and the fact that the HOMO of PEDOT:PSS is typically higher than that of spiro-OMeTAD, reducing the possible $\mathrm{V}_{\mathrm{oc}}$. Additionally, a significant drop in transmission is observed on application of the TLC. Therefore, it can be concluded that although the TLC provides good transparency and hole extraction, it is still not ideal. It can be expected that future developments in perovskitecompatible transparent electrodes will enable even higher PCEs from such semitransparent solar cells.

The TLC is mechanically flexible, meaning that by application of this to such semitransparent solar cells, the first steps towards a roll-to-roll processable semitransparent perovskite solar cell have been taken. If we were to employ a flexible transparent substrate and anode, for example ITO on PET, and combine this with the TLC, it is apparent that such a device could be produced, further enhancing the commercial attractiveness of these solar cells for laminatable semitransparent PV foils.

For a building-integrated photovolatics to perform well all day, they must be effective in harvesting light that is incident at angles other than direct illumination. For the majority of the time, a window will be experiencing illumination angles significantly shallower than direct. As such, we measured the angledependent short-circuit current generated by the $\mathrm{FAPbl}_{3}$ and $\mathrm{MAPbl}_{3}$ semitransparent solar cells, in comparison to monocrystalline and polycrystalline silicon cells. Devices were illuminated with a laser with a small spot size to avoid errors due to variation of total incident power. To also represent power output expected from a working module as a function of angle to the sun, the data was multiplied by $\cos \theta$, to account for the reduced projected footprint of the solar cell with increasing angle. In Figure 5, we show both the current generated from a small spot as a function of angle (solid lines) and multiplied by $\cos \theta$ to represent illumination area larger than the cell (dashed lines). 


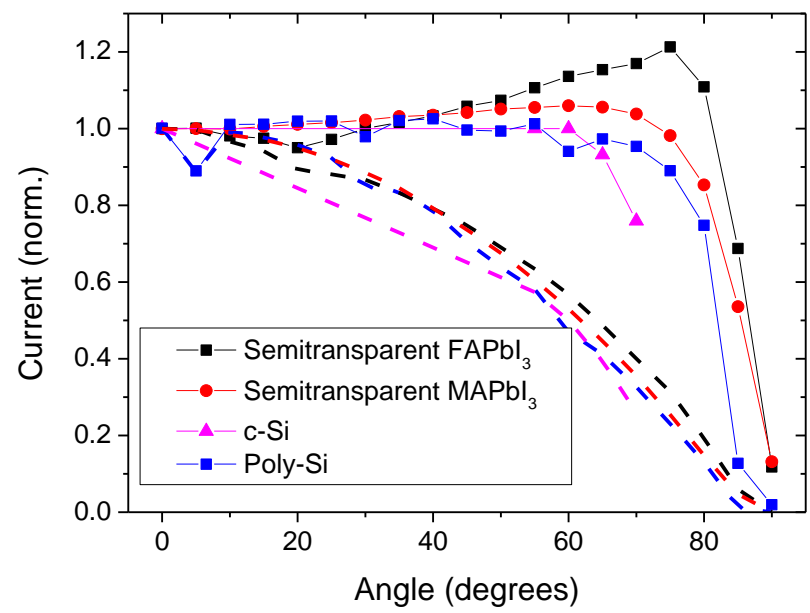

Figure. 5. Angle-dependent photocurrent extracted from $\mathrm{FAPbl}_{3}$ and $\mathrm{MAPbl}_{3}$ semi-transparent solar cells, compared to that extracted from crystalline silicon (c-Si) and polycrystalline silicon (poly-Si) devices. $0^{\circ}$ represents light incident at the normal; light incident at $90^{\circ}$ is coming completely from the side of the device. Dashed lines take into account the reduced footprint of the solar cell with increased illumination angle to represent power output from a module in operation.

We observe that the semi-transparent $\mathrm{FA}$ and $\mathrm{MAPbl}_{3}$ devices performed unprecedentedly well at lower light angles. At angles shallower than the normal, and right up to $\sim 70^{\circ}$ from normal, the $\mathrm{MAPbl}_{3}$ devices generated even more power than they did at the normal. Even more impressively, the $\mathrm{FAPbl}_{3}$ devices show a continuous increase in current generated as the device is angled away from the normal, and generate up to 1.2 times the current extracted from normal incident light at $75 \%$. Notably, both technologies show a drop-off in current at higher angles than either silicon technology tested. c-Si begins to drop in current at $\sim 60^{\circ}$, and polycrystalline silicon at $\sim 65^{\circ}$, as compared to the $M A$ at $70^{\circ}$ and the FA at $80^{\circ}$. We propose that at larger angles of incidence, light will be absorbed by the edges of the perovskite' islands', and can still be converted to current, as opposed to the smooth and flat silicon solar cells. At certain angles, a greater surface area of perovskite will in fact be illuminated due to the aspect 
ratio of the islands, hence the increase above the current displayed at $0^{\circ}$. The particular morphologies of the two perovskites are likely responsible for the distinction between their behaviours.

This demonstrates an important advantage of the semitransparent perovskites, and in particular the $\mathrm{FAPbl}_{3}$, for building-integrated photovoltaics. If this aspect can be enhanced by careful design of the optical properties of each layer, then the semi-transparent perovskite cells may be ideal for BIPV in high irradiance locations, where the sun spends a large fraction of the day close to perpendicular.

The final concern when considering building-integration of solar cells, aside from efficiency, transparency, aesthetics and cost, is that of long-term stability. The methylammonium and formamidinium perovskites suffer from moisture-induced degradation, meaning that effective waterproofing or sealing is necessary for real operation. ${ }^{23}$ For power-generating glass to be a viable installation, it must have a very long functional lifetime to avoid the necessity of replacing windows or window-coatings regularly. Furthermre, for the neutral-coloured application discussed here, one problem could be colour change under moisture degradation, to the yellow of lead iodide, the degradation product. ${ }^{38}$ However, it has been shown recently that when sealed effectively, such devices can be stable for hundreds of hours under full spectrum sunlight. ${ }^{39}$ Additionally, we have recently developed a water-resistant hole-transporting material replacement, which may be able to fully solve the issue of water-sensitivity. ${ }^{38}$ Furthermore, others have demonstrated a screen-printable carbon back electrode/hole transporter configuration that enables long-term moisture stability. ${ }^{40}$ These developments all point towards the possibility of perovskite solar cells that are stable in the long term, even in outdoor environments.

In summary, efficient semi-transparent perovskite solar cells were fabricated by a) incorporating the formamidinium cation into the microstructured architecture and b) application of a novel transparent cathode. The FA-based perovskite displayed higher solar cell performance parameters and lower 
hysteresis than the equivalent methylammonium-based devices, with an efficiency of $6.4 \%$ for a $34 \%$ AVT active layer. With the application of a transparent cathode, we were able to fabricate completely semitransparent full working devices with impressively neutral-coloured transparency. From these, a power conversion efficiency of $5.2 \%$ was achieved from a device with an AVT of $28 \%$. This is a significant improvement on previous reports, rendering the devices very commercially attractive for powergenerating window applications. The angular dependence of the semitransparent perovskite technologies demonstrated excellent performance at non-normal angles, surpassing commercially mature silicon technologies. As such, these solar cells appear to be ideal for all-day operation in a building-integrated setting.

\section{Methods}

Materials. Unless otherwise stated, all materials were purchased from Sigma-Aldrich or Alfa Aesar and used as received. Spiro-OMeTAD was purchased from Borun Chemicals. MAI and FAI were synthesised according to reported procedures. ${ }^{2,23}$

Semi-transparent perovskite solar cell preparation. Devices were fabricated on fluorine-doped tin oxide (FTO) coated glass (Pilkington, $7 \Omega \square^{-1}$ ). Initially FTO was removed from regions under the anode contact by etching the $\mathrm{FTO}$ with $2 \mathrm{M} \mathrm{HCl}$ and zinc powder. Substrates were then cleaned sequentially in hallmanex detergent, acetone, propan-2-ol and oxygen plasma. A $\sim 50 \mathrm{~nm}$ hole-blocking layer of compact TiO2 was deposited by spin-coating a mildly acidic solution of titanium isopropoxide in ethanol (350ul in $5 \mathrm{ml}$ ethanol with $0.013 \mathrm{M} \mathrm{HCl}$ ) at $2000 \mathrm{rpm}$, and annealed at $500^{\circ} \mathrm{C}$ for 30 minutes.

The dewet $\mathrm{FAPbl}_{3}$ perovskite layers were deposited by spin-coating a non-stoichiometric precursor solution of formamidinium iodide, methylammonium iodide and lead chlorine (1:2:1 molar ratio, final concentrations $0.88 \mathrm{M}$ lead chloride, $1.76 \mathrm{M} \mathrm{MAl}$ and $0.88 \mathrm{M}$ FAl) in anhydrous dimethylsulfoxide (DMSO). Spin-coating was carried out at 2000rpm in a nitrogen-filled glovebox. The films were then annealed at $130^{\circ} \mathrm{C}$ for 20 minutes in the glovebox, and then at $170^{\circ} \mathrm{C}$ for 10 minutes in air. 
Dewet $\mathrm{MAPbl}_{3}$ perovskite layers were deposited by spin-coating a non-stoichiometric precursor solution of methylammonium iodide and lead chlorine (3:1 molar ratio, final concentrations $0.88 \mathrm{M}$ lead chloride, 2.64M MAl) in anhydrous dimethylsulfoxide (DMSO). Spin-coating was carried out at 2000rpm in a nitrogen-filled glovebox. The films were then annealed at $130^{\circ} \mathrm{C}$ for 20 minutes in the glovebox.

The hole-transporting layer was then deposited via spin-coating a $0.0788 \mathrm{M}$ solution in chlorobenzene of 2,2',7,7'-tetrakis-(N,N-di-p-methoxyphenylamine)9,9'-spirobifluorene (spiro-OMeTAD), with additives of 0.0184 lithium bis(trifluoromethanesulfonyl)imide (added in $0.61 \mathrm{M}$ acetonitrile solution) and $0.0659 \mathrm{M}$ 4-tert-butylpyridine. Spin-coating was carried out at 2000rpm.

Gold electrodes were thermally evaporated under vacuum of $\sim 10^{-6} \mathrm{Torr}$, at a rate of $\sim 0.1 \mathrm{~nm} / \mathrm{s}$, to complete the devices.

To fabricate the TLC, a commercially available PEDOT:PSS blend (EL-P3145 AGFA) and acrylic microemulsion pressure sensitive adhesive (F46 Styccobond) were mixed such that the final volume fraction of the PEDOT:PSS in the dry film was 0.0175 . This corresponded to a $1: 1.34$ Styccobond:PEDOT:PSS ratio by weight. This mix was then tape cast onto sheets of commercially available flexible microgrid sheets (Epimesh 300 from Epigem) at a thickness of $\sim 90 \mu \mathrm{m}$. The films were dried for 15 minutes at $60{ }^{\circ} \mathrm{C}$ followed by 5 minutes at $120{ }^{\circ} \mathrm{C}$ leaving final dried TCA films of $\sim 30 \mu \mathrm{m}$ thick. Cells to which the TLC was to be applied had a solution of PEDOT:PSS (Hereaus GSD1330S) mixed with ethanol and isopropanol in a 1:1:1 ratio by volume sprayed onto the HTL surface whilst the cell was held on a $50^{\circ} \mathrm{C}$ hotplate and left for 10 seconds post spraying. This left a layer of PEDOT:PSS approximately $50 \mathrm{~nm}$ thick. The TLC fabricated as described above was then laminated onto the PEDOT:PSS surface of the cells using finger pressure.

Device Characterisation. The current density-voltage (J-V) curves were measured (2400 Series SourceMeter, Keithley Instruments) under simulated AM 1.5 sunlight at $100 \mathrm{mWcm}^{-2}$ irradiance generated by an Abet Class AAB sun 2000 simulator, with the intensity calibrated with an NREL 
calibrated KG5 filtered Si reference cell. The mismatch factor was calculated to be less than $1 \%$. The solar cells were masked with a metal aperture to define the active area of $0.0625 \mathrm{~cm}^{-2}$ and measured in a light-tight sample holder to minimize any edge effects and ensure that the reference cell and test cell are located during measurement in the same spot under the solar simulator. For the fast JV scans, ells were scanned from forwards bias to short-circuit at a rate of $0.38 \mathrm{~V} / \mathrm{s}$ after holding under illumination at $1.4 \mathrm{~V}$ for 5 seconds. Maximum power point was determined from these fast $\mathrm{JV}$ scans and current measured holding at this voltage for the maximum power point holding scans.

Angle-dependent photocurrent. Angle-dependent photocurrent was measured by carrying out J-V sweeps with a Keithley 2636 SourceMeter upon illumination of the device with a $532 \mathrm{~nm}$ CW laser, spot size $0.1 \mathrm{~cm}^{2}$, with the beam appropriately attenuated to produce similar currents as when the devices were under AM1.5 solar simulated illumination. Extra-large gold electrodes were evaporated onto devices fabricated as described previously. The spot was kept within the bounds of the device region at all times, and the device was rotated on a calibrated stage, taking measurements every $5^{\circ} .3$ or more devices were measured to test reproducibility. Silicon solar cells were purchased from PV Measurements.

External Quantum Efficiency (EQE) measurements were performed via Fourier transform photocurrent spectroscopy (FTPCS). Briefly, the devices were place in the sample comparment of a Bruker Vertex 80 Fourier transform spectrometer (FTIR) with tungsten-halogen light source and $\mathrm{CaF}_{2}$ beam-splitter. The photocurrent from the device under test was collected by a transimpedance amplifier (SRS570) before being fed into the analogue to digital convertor of the FTIR. Custom control electronics and software allow the device to be biased at $1.4 \mathrm{~V}$ prior to measuring the device in short circuit configuration. After each set of measurements the FTPCS spectrum of a Newport calibrated silicon solar cell with BK7 windows was measured to allow absolute EQE values to be determined. 
Optical measurements. Transmittance and reflectance spectra were collected with a Varian Cary 300 UV-Vis spectrophotometer with an internally coupled integrating sphere.

Film characterisation: A Hitachi S-4300 field emission scanning electron microscope was used to acquire SEM images. Sample thicknesses were measured using a Veeco Dektak 150 surface profileometer.

\section{Supporting Information}

Supporting information contains device statistics and J-V hysteresis scan data. This material is available free of charge via the Internet at http://pubs.acs.org.

\section{Acknowledgements}

This work was supported by EPSRC and Oxford Photovoltaics Ltd. through a Nanotechnology KTN CASE award, and the European Research Council (ERC) HYPER PROJECT no. 279881.

\section{References}

(1) Stranks, S. D.; Eperon, G. E.; Grancini, G.; Menelaou, C.; Alcocer, M. J. P.; Leijtens, T.; Herz, L. M.; Petrozza, A.; Snaith, H. J. Electron-Hole Diffusion Lengths Exceeding 1 Micron in an Organometal Trihalide Perovskite Absorber. Science 2013, 342, 341-344.

(2) Lee, M. M.; Teuscher, J.; Miyasaka, T.; Murakami, T. N.; Snaith, H. J. Efficient Hybrid Solar Cells Based on Meso-Superstructured Organometal Halide Perovskites. Science 2012, 338, 643-647.

(3) Kim, H.-S.; Lee, C.-R.; Im, J.-H.; Lee, K.-B.; Moehl, T.; Marchioro, A.; Moon, S.-J.; Humphry-Baker, R.; Yum, J.-H.; Moser, J. E.; et al. Lead lodide Perovskite Sensitized All-Solid-State Submicron Thin Film Mesoscopic Solar Cell with Efficiency Exceeding 9\%. Sci. Rep. 2012, 2, 591:1-7.

(4) Xing, G.; Mathews, N.; Lim, S. S.; Lam, Y. M.; Mhaisalkar, S.; Sum, T. C. Long-Range Balanced Electron- and Hole-Transport Lengths in Organic-Inorganic $\mathrm{CH}_{3} \mathrm{NH}_{3} \mathrm{Pbl}_{3} .2013,6960,498-500$.

(5) Kojima, A.; Teshima, K.; Shirai, Y.; Miyasaka, T. Organometal Halide Perovskites as Visible-Light Sensitizers for Photovoltaic Cells. J. Am. Chem. Soc. 2009, 131, 6050-6051.

(6) Jeon, N. J.; Lee, H. G.; Kim, Y. C.; Seo, J.; Noh, J. H.; Lee, J.; Seok, S. II. O-Methoxy Substituents in Spiro-OMeTAD for Efficient Inorganic-Organic Hybrid Perovskite Solar Cells. J. Am. Chem. Soc. 2014, 136, 7837-7840. 
(7) Lee, J.-W.; Seol, D.-J.; Cho, A.-N.; Park, N.-G. High-Efficiency Perovskite Solar Cells Based on the Black Polymorph of $\mathrm{HC}\left(\mathrm{NH}_{2}\right)_{2} \mathrm{Pbl}_{3}$. Adv. Mater. 2014, 6, 4991-4998.

(8) Zhou, H.; Chen, Q.; Li, G.; Luo, S.; Song, T. -b.; Duan, H.-S.; Hong, Z.; You, J.; Liu, Y.; Yang, Y. Interface Engineering of Highly Efficient Perovskite Solar Cells. Science 2014, 345, 542-546.

(9) Jeon, N. J.; Noh, J. H.; Kim, Y. C.; Yang, W. S.; Ryu, S.; Seok, S. Il. Solvent Engineering for HighPerformance Inorganic-Organic Hybrid Perovskite Solar Cells. Nat. Mater. 2014, 13, 897-903.

(10) Eperon, G. E.; Burlakov, V. M.; Goriely, A.; Snaith, H. J. Neutral Color Semitransparent Microstructured Perovskite Solar Cells. ACS Nano 2014, 8, 591-598.

(11) Roldan, C.; Malinkiewicz, O.; Betancur, R.; Longo, G.; Momblona, C.; Jaramillo, F.; Camacho, L.; Bolink, H. J. High Efficiency Single-Junction Semitransparent Perovskite Solar Cells. Energy Environ. Sci. 2014, 7, 2968-2973.

(12) Manser, J. S.; Kamat, P. V. Band Filling with Free Charge Carriers in Organometal Halide Perovskites. Nat. Photonics 2014, 8, 737-743.

(13) D'Innocenzo, V.; Grancini, G.; Alcocer, M. J. P.; Kandada, A. R. S.; Stranks, S. D.; Lee, M. M.; Lanzani, G.; Snaith, H. J.; Petrozza, A. Excitons versus Free Charges in Organo-Lead Tri-Halide Perovskites. Nat. Commun. 2014, 5, 1-6.

(14) Deschler, F.; Price, M.; Pathak, S.; Klintberg, L. E.; Jarausch, D.-D.; Higler, R.; Hüttner, S.; Leijtens, T.; Stranks, S. D.; Snaith, H. J.; et al. High Photoluminescence Efficiency and Optically Pumped Lasing in Solution-Processed Mixed Halide Perovskite Semiconductors. J. Phys. Chem. Lett. 2014, 5, 1421-1426.

(15) Chueh, C.-C.; Chien, S.-C.; Yip, H.-L.; Salinas, J. F.; Li, C.-Z.; Chen, K.-S.; Chen, F.-C.; Chen, W.-C.; Jen, A. K.-Y. Toward High-Performance Semi-Transparent Polymer Solar Cells: Optimization of Ultra-Thin Light Absorbing Layer and Transparent Cathode Architecture. Adv. Energy Mater. 2013, 3, 417-423.

(16) Yusoff, A. R. B. M.; Lee, S. J.; Shneider, F. K.; da Silva, W. J.; Jang, J. High-Performance Semitransparent Tandem Solar Cell of 8.02\% Conversion Efficiency with Solution-Processed Graphene Mesh and Laminated Ag Nanowire Top Electrodes. Adv. Energy Mater. 2014, 4, 1301989:1-10.

(17) Chang, C.-Y.; Zuo, L.; Yip, H.-L.; Li, C.-Z.; Li, Y.; Hsu, C.-S.; Cheng, Y.-J.; Chen, H.; Jen, A. K.-Y. Highly Efficient Polymer Tandem Cells and Semitransparent Cells for Solar Energy. Adv. Energy Mater. 2014, 4, 1301645:1-6.

(18) Docampo, P.; Hanusch, F.; Stranks, S. D.; Döblinger, M.; Feckl, J. M.; Ehrensperger, M.; Minar, N. K.; Johnston, M. B.; Snaith, H. J.; Bein, T. Solution Deposition-Conversion for Planar Heterojunction Mixed Halide Perovskite Solar Cells. Adv. Energy Mater. 2014, 1400355:1-6. 
(19) You, J.; Hong, Z.; Yang, Y. M.; Chen, Q.; Cai, M.; Song, T.; Chen, C.; Lu, S.; Liu, Y.; Zhou, H.; et al. Low-Temperature Solution-Processed Perovskite Solar Cells with High Efficiency and Flexibility. ACS Nano 2014, 8, 1674-1680.

(20) Zhao, Y.; Zhu, K. CH 3 NH 3 Cl-Assisted One-Step Solution Growth of CH 3 NH 3 Pbl 3 : Structure, Charge-Carrier Dynamics, and Photovoltaic Properties of Perovskite Solar Cells. J. Phys. Chem. C 2014, 118, 9412-9418.

(21) Colella, S.; Mosconi, E.; Fedeli, P.; Listorti, A.; Orlandi, F.; Ferro, P.; Besagni, T.; Rizzo, A.; Calestani, G.; Gigli, G.; et al. MAPbI3-xClx Mixed Halide Perovskite for Hybrid Solar Cells : The Role of Chloride as Dopant on the Transport and Structural Properties. Chem. Mater. 2013, 25, 4613-4618.

(22) Grancini, G.; Marras, S.; Prato, M.; Giannini, C.; Quarti, C.; De Angelis, F.; De Bastiani, M.; Eperon, G. E.; Snaith, H. J.; Manna, L.; et al. The Impact of the Crystallization Processes on the Structural and Optical Properties of Hybrid Perovskite Films for Photovoltaics. J. Phys. Chem. Lett. 2014, 140926103112004.

(23) Eperon, G. E.; Stranks, S. D.; Menelaou, C.; Johnston, M. B.; Herz, L. M.; Snaith, H. J. Formamidinium Lead Trihalide: A Broadly Tunable Perovskite for Efficient Planar Heterojunction Solar Cells. Energy Environ. Sci. 2014, 7, 982.

(24) Pellet, N.; Gao, P.; Gregori, G.; Yang, T.-Y.; Nazeeruddin, M. K.; Maier, J.; Grätzel, M. MixedOrganic-Cation Perovskite Photovoltaics for Enhanced Solar-Light Harvesting. Angew. Chem. Int. Ed. Engl. 2014, 53, 3151-3157.

(25) Burlakov, V. M.; Eperon, G. E.; Snaith, H. J.; Chapman, S. J.; Goriely, A. Controlling Coverage of Solution Cast Materials with Unfavourable Surface Interactions. Appl. Phys. Lett. 2014, 104, 091602.

(26) Dualeh, A.; Tétreault, N.; Moehl, T.; Gao, P.; Nazeeruddin, M. K.; Grätzel, M. Effect of Annealing Temperature on Film Morphology of Organic-Inorganic Hybrid Pervoskite Solid-State Solar Cells. Adv. Funct. Mater. 2014, 24, 3250-3258.

(27) Eperon, G. E.; Burlakov, V. M.; Docampo, P.; Goriely, A.; Snaith, H. J. Morphological Control for High Performance, Solution-Processed Planar Heterojunction Perovskite Solar Cells. Adv. Funct. Mater. 2014, 24, 151-157.

(28) Stoumpos, C. C.; Malliakas, C. D.; Kanatzidis, M. G. Semiconducting Tin and Lead lodide Perovskites with Organic Cations: Phase Transitions, High Mobilities, and Near-Infrared Photoluminescent Properties. Inorg. Chem. 2013, 52, 9019-9038.

(29) Koh, T. M.; Fu, K.; Fang, Y.; Chen, S.; Sum, T. C.; Mathews, N.; Mhaisalkar, S. G.; Boix, P. P.; Baikie, T. Formamidinium-Containing Metal-Halide: An Alternative Material for Near-IR Absorption Perovskite Solar Cells. J. Phys. Chem. C 2013, 118, 16458-16462. 
(30) Snaith, H. J.; Abate, A.; Ball, J. M.; Eperon, G. E.; Leijtens, T.; Noel, N. K.; Stranks, S. D.; Wang, J. T.; Wojciechowski, K.; Zhang, W. Anomalous Hysteresis in Perovskite Solar Cells. J. Phys. Chem. Lett. 2014, 5, 1511-1515.

(31) Bryant, D.; Greenwood, P.; Troughton, J.; Wijdekop, M.; Carnie, M.; Davies, M.; Wojciechowski, K.; Snaith, H. J.; Watson, T.; Worsley, D. A Transparent Conductive Adhesive Laminate Electrode for High-Efficiency Organic-Inorganic Lead Halide Perovskite Solar Cells. Adv. Mater. 2014, 26, 7499-7504.

(32) Chen, K.-S.; Salinas, J.-F.; Yip, H.-L.; Huo, L.; Hou, J.; Jen, A. K.-Y. Semi-Transparent Polymer Solar Cells with 6\% PCE, 25\% Average Visible Transmittance and a Color Rendering Index close to 100 for Power Generating Window Applications. Energy Environ. Sci. 2012, 5, 9551-9557.

(33) Snaith, H. J. Perovskites: The Emergence of a New Era for Low-Cost, High-Efficiency Solar Cells. J. Phys. Chem. Lett. 2013, 4, 3623-3630.

(34) Conings, B.; Baeten, L.; De Dobbelaere, C.; D’Haen, J.; Manca, J.; Boyen, H.-G. Perovskite-Based Hybrid Solar Cells Exceeding 10\% Efficiency with High Reproducibility Using a Thin Film Sandwich Approach. Adv. Mater. 2014, 26, 2041-2046.

(35) Subbiah, A. S.; Halder, A.; Ghosh, S.; Mahuli, N.; Hodes, G.; Sarkar, S. K. Inorganic Hole Conducting Layers for Perovskite-Based Solar Cells. J. Phys. Chem. Lett. 2014, 5, 1748-1753.

(36) Liu, D.; Kelly, T. L. Perovskite Solar Cells with a Planar Heterojunction Structure Prepared Using Room-Temperature Solution Processing Techniques. Nat. Photonics 2013, 8, 133-138.

(37) Wojciechowski, K.; Saliba, M.; Leijtens, T.; Abate, A.; Snaith, H. J. Sub- $150{ }^{\circ} \mathrm{C}$ Processed MesoSuperstructured Perovskite Solar Cells with Enhanced Efficiency. Energy Environ. Sci. 2014, 7, 1142.

(38) Habisreutinger, S. N.; Leijtens, T.; Eperon, G. E.; Stranks, S. D.; Nicholas, R. J.; Snaith, H. J. Carbon Nanotube/polymer Composite as a Highly Stable Charge Collection Layer in Perovskite Solar Cells. Nano Lett. 2014, 14, 5561-5568.

(39) Leijtens, T.; Eperon, G. E.; Pathak, S.; Abate, A.; Lee, M. M.; Snaith, H. J. Overcoming Ultraviolet Light Instability of Sensitized TiO2 with Meso-Superstructured Organometal Trihalide Perovskite Solar Cells. Nat. Commun. 2013, 4, 2885.

(40) Mei, a.; Li, X.; Liu, L.; Ku, Z.; Liu, T.; Rong, Y.; Xu, M.; Hu, M.; Chen, J.; Yang, Y.; et al. A HoleConductor-Free, Fully Printable Mesoscopic Perovskite Solar Cell with High Stability. Science 2014, 345, 295-298. 